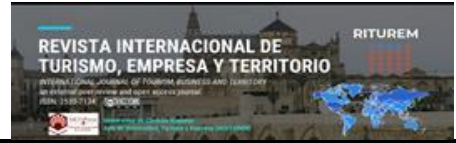

Cita bibliográfica: Corzo Arévalo, D. (2020). La gestion de departamentos y destinos turísticos de Colombia según el Índice de Competitividad Turística Regional. Revista Internacional de Turismo, Empresa y Territorio, 4 (2), 158-177. https://doi.org/10.21071/riturem.v4i2.13010

\title{
La gestión de departamentos y destinos turísticos de Colombia según el Índice de Competitividad Turística Regional (ICTRC)
}

\section{Tourist departments and destinations of Colombia according to the Regional Tourist Competitiveness Index (ICTRC)}

\author{
Daniel Corzo Arévalo 1 ${ }^{*}$ \\ Sebastián García Méndez ${ }^{2}$
}

\section{Resumen}

El boom turístico de las últimas décadas ha motivado a los gobiernos a enfocarse en impulsar la competitividad turística de sus países y destinos, como un catalizador del desarrollo humano. La presente investigación realiza un análisis comparativo de las diversas variables del Índice de Competitividad Turística Regional de Colombia (ICTRC) desarrollado por el Centro de Pensamiento Turístico (CPTUR), inherentes a la dimensión de Gestión del Destino en 22 territorios de Colombia. Se aplicó el método del análisis de componentes principales para medir la correlación entre 18 variables seleccionadas del ICTRC, que dan cuenta de la gestión de destinos en Colombia. Se identificó que, para mejorar la competitividad turística de los destinos en Colombia, los focos principales deben orientarse hacia la información turística, los eventos terroristas, los niveles de presupuestales, el retorno a la contribución parafiscal y la inclusión del turismo en los planes de desarrollo. Se recomienda que las entidades gubernamentales formulen, implementen y validen procesos a nivel macro y micro en los constructos referidos, con el fin de impactar considerablemente la competitividad turística de los destinos.

Palabras clave: Competitividad turística; destinos turísticos; análisis de componentes principales; Colombia.

\begin{abstract}
In recent decades, the tourism boom has motivated governments to focus on boosting the tourism competitiveness of their countries and destinations, as a catalyst for human development. This research

\footnotetext{
${ }^{1}$ Docente investigador Grupo de investigación GECAC, Corporación Universitaria de Ciencia y Desarrollo UNICIENCIA. Bucaramanga, Colombia. E-mail: dcorzoarevalo@unicienciabga.edu.co Id orcid: http://orcid.org/0000-0001-6017-8028* Autor para la correspondencia

2 Docente investigador Grupo de investigación SINERGIA, Universidad de Investigación y Desarrollo. Bucaramanga, Colombia. E-mail: sgarcia15@udi.edu.co Id orcid: http://orcid.org/0000-0002-6077-0248
} 
performs a comparative analysis of the various variables from the Regional Tourism Competitiveness Index of Colombia (ICTRC), developed by the Centre for Tourism Thinking (CPTUR), within the Destination Management dimension in 22 departments of Colombia. The principal components analysis method was applied to measure the correlation between 18 variables selected from the ICTRC, which provide a measurable account of the efficacy of destination management in Colombia. It was identified that, to improve tourist destination competitiveness in Colombia, the main focus should be on tourist information, terrorist events, budget levels, the return to the parafiscal contribution and the inclusion of tourism in development plans. It is recommended that government entities formulate, implement and validate processes at the macro and micro level in the aforementioned constructs, in order to considerably impact tourist destination competitiveness.

Keywords: Tourist competitiveness; touristic destinations; principal component analysis; Colombia.

\section{Introducción}

En las últimas décadas, el turismo ha venido ganando protagonismo como un sector esencial en la economía mundial y un catalizador del desarrollo humano a nivel global, permitiendo a los países diversificar su economía y mejorar sus tasas de empleo, ingresos y su balanza de pagos, impulsando con ello la transformación de sus condiciones socioculturales y económicas (Das \& Dirienzo, 2009; Das \& Dirienzo, 2012; Croes \& Kubickova, 2013; Mihalic \& Aramberri, 2015; Azzopardi \& Nash, 2017; Nazmfar, Eshghei, Alavi \& Pourmoradian, 2019; Wang \& Liu, 2020).

Este boom ya ha alcanzado nuevos territorios que se han convertido en nuevos destinos turísticos y hoy compiten con los destinos tradicionales (Rodríguez \& Pulido, 2020), creando enormes oportunidades para el bienestar de las personas, pero también desafíos y amenazas a las comunidades y el medio ambiente circundante (Hermawati, 2020). El rápido desarrollo del sector promueve no sólo la rivalidad entre competidores, sino también la cooperación, mientras buscan conjuntamente las mejores soluciones a sus problemas (Maráková, Dyr \& WolakTuzimek, 2016).

En países en los que el sector del turismo se ha vuelto un motor económico vital, las autoridades gubernamentales han centrado su atención en los factores que podrían darles una ventaja competitiva (Isa, Ariyanto, \& Kiumarsi, 2020). Con ello, la competitividad turística no solo se convierte en un indicador importante para evaluar la rentabilidad del sector turístico en un país (Serrano, Montoya \& Cazares, 2018), sino en objeto de estudio de diversos investigadores alrededor de todo el mundo, en tal sentido, la presente investigación realiza un análisis comparativo de las diversas variables inherentes a la dimensión de Gestión del Destino en 22 Departamentos de Colombia.

En este contexto, el documento se estructuró de la siguiente forma: en el apartado siguiente se definen los conceptos de competitividad turística, competitividad en destinos turísticos e índice de competitividad turística. En el tercer apartado se expone el método y las diversas variables concernientes a la gestión de destinos de 22 zonas geográficas de Colombia (departamentos) en el periodo 2017-2019. Posteriormente se muestran los resultados del análisis de componentes principales, midiendo la correlación entre 18 variables seleccionadas 
del ICTRT, que dan cuenta de la gestión de destinos en Colombia. Finalmente, se presentan las conclusiones obtenidas en el estudio.

\section{Fundamentación teórica}

\subsection{Competitividad turística}

En países turísticos desarrollados y menos desarrollados, la competitividad turística es una cuestión clave para los gestores de las políticas públicas dirigidas al desarrollo del turismo (Krstic, Jovanovic, Jankovic-Milic \& Stanisic, 2016). Ahora bien, dado que la actividad turística representa tantas oportunidades como amenazas, la estrategia competitiva del sector debe estar alineada con los principios de desarrollo sostenible.

Kubickova \& Lee (2018) proponen que la competitividad turística se puede evaluar desde cuatro componentes distintos: (a) capacidad de desplegar recursos (sostenibilidad), (b) experiencias turísticas memorables, (c) rendimiento superior y (d) calidad de vida. Por otro lado, Croes, Ridderstaat \& Shapoval (2020) la reducen a dos componentes: satisfacción y productividad. La satisfacción determina está asociada con la capacidad del destino de entregar valor a los turistas a través de experiencias memorables - el punto b de Kubickova \& Lee (2018). Mientras tanto, la productividad se relaciona directamente con el desarrollo humanopuntos a, c y d de Kubickova \& Lee (2018) — y determina en qué medida se usan eficazmente los recursos, se crea valor en su utilización y se mide su rendimiento. Todos estos autores coinciden en abordar la competitividad turística desde un enfoque de desarrollo humano, promoviendo la utilización de los recursos para proporcionar beneficios económicos, sociales y ambientales que superen sus costos correspondientes.

En términos conceptuales puede hacerse alusión en el marco de la posibilidad de prestar servicios turísticos con ingresos superiores a la competencia, basado en diversas ventajas competitivas que le brindan un valor diferenciado (Wan, Yan, Wang, Liu, Wang \& Wang, 2019). Esta capacidad puede, a su vez, entenderse desde tres niveles: (1) el nivel del país (competitividad de la producción turística), (2) el nivel del destino o (3) el nivel de la empresa turística (Benavides \& Venegas, 2020).

$\mathrm{Si}$ bien es necesario contar con una mejor oferta turística para incrementar la competitividad, ésta no puede reducirse a medir y comparar los flujos turísticos que alcanzan los entes receptores (Zuñiga \& Castillo, 2012), puesto que se debe brindar especial relevancia a la capacidad de sostener e incrementar el posicionamiento en mercados externos, y el mejoramiento de las condiciones de vida de la población. En esta línea, las premisas orientadoras se enmarcan en la eficiencia macroeconómico, las políticas gubernamentales y el atractivo para los inversionistas en términos de empleo y crecimiento económico local (González \& Mendieta, 2009).

Por otro lado, la competitividad turística a nivel empresarial relaciona el posicionamiento en los mercados, los beneficios y la expansión de operaciones (Perles, Ramón y Sevilla, 2014). Esta capacidad depende de cinco fuerzas competitivas, las refieren los clientes, los proveedores, los nuevos entrantes, los productos o servicios sustitutos y la competencia del sector. (Porter, 1985). 


\subsection{La competitividad de los destinos turísticos}

El destino es el territorio en el que confluyen elementos y recursos físicos que constituyen atractivos turísticos, como las montañas, los hoteles, las edificaciones y la tecnología, que se combinan con los procesos de planeación, gestión e imagen, para dar lugar a productos turísticos determinados que conforman la experiencia de la visita (Corzo, 2013). De igual manera, el gasto turístico, la macroeconomía, la gobernabilidad, las políticas y los hechos internacionales que generan atracción o aversión al destino. En el caso del turismo, la oferta no puede desligarse de la ubicación y contexto geográfico donde se encuentra.

La concepción más básica de competitividad propone que un destino turístico es competitivo si su crecimiento económico y el desarrollo de sus recursos pueden asociarse con un aumento del flujo y gasto turístico, que permiten comparar las posiciones relativas de los diversos destinos (Dogru, Suess \& Sirakaya-Turk, 2020; Mustafa, Omar \& Mukhiar, 2020). Es decir, la competitividad de un destino turístico puede medirse en términos de su efectividad para atraer y satisfacer a los visitantes a través de la calidad y las características de la experiencia turística (Brandão, Joia \& do Canto Cavalheiro, 2019; Benavides \& Venegas, 2020). La lógica que subyace tras esta visión de la competitividad de destinos turísticos es que un destino es más competitivo cuando atrae a más visitantes, que gastan más dinero en el destino, lo que lleva a un aumento del PIB y crecimiento económico, que a su vez se traducen en un mayor bienestar de la población local (Webster \& Ivanov, 2014).

En este contexto, Hong (2009) define la competitividad turística en términos de crear, integrar y ofrecer experiencias turísticas transversales, es decir, contemplando los diferentes bienes y servicios, contemplando la satisfacción de los turistas, generando los recursos de un destino y le ayudan a mantener una posición de ventaja en el mercado, en relación con otros destinos. Cabe mencionar que, si bien la disponibilidad de recursos puede impulsar la competitividad del destino y colocarlo en una posición diferenciada en el mercado, los recursos aportan poco si no se gestionan adecuadamente para transformarlos en productos turísticos (Andrades \& Dimanche, 2017).

Desde el enfoque de desarrollo sostenible, esta dimensión económica y objetiva sólo es una parte de la figura, que se complementa con una dimensión no económica y subjetiva (Knežević, Dwyer, Koman \& Mihalič, 2016). Bajo esta perspectiva más holística, se puede decir que la competitividad de un destino turístico está relacionada con su capacidad para conseguir un diverso grupo de objetivos - algunos asociados con la demanda, junto con otros más amplios, los cuales vinculan aspectos de la economía, la sociedad y el medioambiente (Perles, Ramón y Sevilla, 2014). En años recientes, la competitividad ha venido incorporando los componentes legales, siendo estos promotores a priori de productividad, cadenas de valor y clusters. Actualmente, se reconoce que la competitividad de un destino turístico depende de las sinergias de un conjunto de factores, tales como organizaciones, infraestructura, recursos humanos, entorno e institucionalidad (Acerenza, 2009). 


\section{3. Índice de Competitividad Turística}

Dada la subjetividad de la competitividad turística, la multiplicidad de variables que intervienen en su evaluación y la dificultad de dibujar los límites entre un destino y su entorno, resulta difícil formular medidas reales de competitividad basadas en datos cuantitativos o cualitativos (Crouch, 2011; Garcés, Sarmiento \& Paneca 2018). Aunque existen abundantes fuentes de datos, como el flujo de visitantes, ingresos turísticos, pernoctaciones, etc., no hay consenso en determinar qué variables son medidas apropiadas de la competitividad, pues muchas de ellas reflejan, a lo sumo, el volumen de la demanda turística (Perles, 2016). Inclusive, se ha visto que los estudios que utilizan la cuota de mercado como indicador de competitividad turística, que en una época alcanzaron gran popularidad, pueden orientar el enfoque de las políticas de crecimiento a maximizar esta variable, comprometiendo la sostenibilidad económico-ambiental de los destinos que cuentan con recursos limitados.

Aun así, se ha desarrollado una diversidad de modelos para medir la competitividad de un destino, tales como el postulado por Porter, integrando elementos económicos e industriales, el formulado por el Instituto Alemán; el modelo de Crouch y Ritchie; el referido por Dwyer y Kim; el vinculable a Heath; e índices propuestos por el Foro Económico Mundial (FEM) y el Ministerio de Comercio, Industria y Turismo (Corzo, 2013).

Entre los modelos académicos, el modelo de Ritchie y Crouch (2010) es uno de los más difundidos para medir la competitividad y la sostenibilidad de los destinos en la literatura. Se compone de siete elementos: el entorno global (macro); el entorno competitivo (micro); los recursos básicos y los atractivos; factores y recursos de apoyo; política, planificación y desarrollo de los destinos; gestión del destino; calificación y amplificación de los factores determinantes (Kolosinska, Petrashchak, Kolosinskyi \& Katana, 2018). Así también, Gooroochurn \& Sugiyarto (2004) propusieron un modelo para evaluar la competitividad de los destinos turísticos, basado en ocho factores: (1) precios; (2) apertura económica; (3) tecnología; (4) estructura; (5) desarrollo humano en el ámbito del turismo; (6) desarrollo social; (7) medio ambiente y (8) recursos humanos.

El Foro Económico Mundial es la entidad que aporta algunos de los índices más reconocidos a nivel global para medir la competitividad. Entre ellos, el Índice de Competitividad de Viajes y Turismo (TTCI) del Foro Económico Mundial (FEM) es el más común para medir la competitividad del turismo (Rodríguez \& Pulido, 2020a). El TTCI se publica anualmente en el Global Competitiveness Report, un compendio de los indicadores de competitividad económica de 139 países elaborado por el FEM (Rodríguez \& Pulido, 2020). Más que un modelo de competitividad turística, el TTCI es una herramienta de benchmarking que se utiliza en la formulación de políticas y estrategias enfocadas en promover la sustentabilidad de un destino a largo plazo (Ortiz, Robles \& Guzmá, 2018).

Basándose en el TTCI del FEM, la Universidad Autónoma de Madrid propuso un modelo cuantitativo objetivo de competitividad de los países como destinos turísticos (Rodríguez, Alonso \& Celerin, 2016), el cual integra diversa información analizada bajo criterios estadísticos provenientes actores públicos y privados, siendo aplicado por primera vez en la Unión Europea. 
En Colombia específicamente, el Ministerio de Comercio, Industria y Turismo publicó en 2008 el Sistema de Indicadores de Competitividad para el sector turístico (MINCIT, 2008). Esta guía aportó una batería de indicadores que fueron aplicados por varias regiones durante los años posteriores, pero no sirvió como referente de análisis estadístico ya que pocos destinos lo aplicaron-los resultados pierden sentido si no se comparan relativamente con los resultados del mismo sistema aplicado en otras regiones (Varela \& Olarte, 2018). Desde 2016, el Centro de Pensamiento Turístico, instancia de investigación creada por la Asociación Hotelera y Turística de Colombia (Cotelco), en asociación con la Fundación Universitaria Cafam, vienen publicando el Índice de Competitividad Turística Regional de Colombia (ICTRC) de todos los departamentos del país, dando respuesta al problema de su limitada aplicación y facilitando su aprovechamiento por parte de los gestores públicos, la academia y la sociedad en general (CPTUR, 2016).

\section{Metodología}

El estudio se estructuró bajo un enfoque cuantitativo y tuvo un diseño no experimental de corte transversal, en tal sentido, es de tipo correlacional, ya que el interés se centra en conocer la relación que existe entre dos o más conceptos, particularmente variables de la dimensión de la competitividad turística orientada a la Gestión del Destino (Hernández, Fernández \& Baptista, 2006).

La población y muestra de la presente investigación fueron 22 zonas geográficas (Departamentos) objeto de estudio en el Índice de Competitividad Turística Regional de Colombia (ICTRC) desarrollado por el Centro de Pensamiento Turístico - Colombia en el plano temporal 2017 - 2019, los cuales se presentan la siguiente Tabla.

Tabla 1. Zonas geográficas objeto de estudio

\begin{tabular}{|c|c|c|c|}
\hline Antioquia & Caquetá & Huila & Risaralda \\
\hline Atlántico & Casanare & Magdalena & Santander \\
\hline Bogotá D.C. & Cauca & Meta & Tolima \\
\hline Bolívar & Cesar & Nariño & Valle del Cauca \\
\hline Boyacá & Córdoba & Norte de Santander & \\
\hline Caldas & Cundinamarca & Quindío & \\
\hline
\end{tabular}

Fuente: Elaboración propia

El procesamiento de la información se realizó por medio del análisis descriptivo, correlacional y de componentes principales, siendo esta última una técnica multivariante para la reducción de datos (Pérez et al, 2009) y propender una mejor interpretación (Peña, 2002). En esta línea, los indicadores objeto de estudio se presentan en la siguiente Tabla 2. 
Tabla 2. Data objeto de estudio

\begin{tabular}{|l|c|}
\hline \multicolumn{1}{|c|}{ NOMBRE DE LA VARIABLE } & CODIFICACIÓN \\
\hline Destinos certificados en sostenibilidad & GES1 \\
\hline Planes de desarrollo turístico & GES4 \\
\hline Turismo en los planes de desarrollo & GES5 \\
\hline Contribución parafiscal & GES9 \\
\hline Financiamiento de proyecto & GES10 \\
\hline Presupuesto de inversión destinado al turismo & GES15 \\
\hline Oficinas especializadas en gestión turística & GES19 \\
\hline Producción de información estadística de viajes y turismo & GES29 \\
\hline Seguridad de empresas turísticas & GES32 \\
\hline Eventos terroristas & GES33 \\
\hline Homicidios & GES34 \\
\hline Disponibilidad policial & GES36 \\
\hline Disponibilidad policía de turismo & GES37 \\
\hline Puntos de información turística & GES38 \\
\hline Accidentalidad vial & GES40 \\
\hline Retorno a la contribución parafiscal & GES43 \\
\hline Presupuesto regionalizado para el turismo & GES44 \\
\hline Delitos cometidos contra turistas & GES45 \\
\hline
\end{tabular}

Fuente: Elaboración propia

\section{Resultados y Discusión}

\subsection{Análisis descriptivo}

Los estadísticos descriptivos del presente estudio se centran en el análisis comparativo de las diversas variables inherentes a la dimensión de Gestión del Destino del Índice de Competitividad Turística Regional desarrollado por el Centro de Pensamiento Turístico Colombia en el periodo temporal 2017 - 2019, en tal sentido, se tuvieron presentes las zonas geográficas estudiadas en las tres versiones, con el propósito de generar un idóneo análisis.

La Tabla 3 relaciona la media de los constructos y el porcentaje de zonas geográficas con puntuaciones iguales o superiores a la métrica descrita, así mismo, es válido mencionar que a nivel macro se constituyen 3 conglomerados planteados por el ente emisor, contemplando como base de inclusión o exclusión el aporte del PIB, siendo esto alineado a lo postulado por Romão (2020) en torno al arraigo del turismo en los territorios y Cavalheiro et al. (2020) referente a elementos vinculables a la planificación. 
Tabla 3. Estadísticos descriptivos Gestión del destino. Periodo 2017 - 2019

\begin{tabular}{|l|c|c|c|c|c|c|}
\hline \multirow{2}{*}{ Variable } & \multicolumn{2}{|c|}{2017} & \multicolumn{2}{c|}{$\mathbf{2 0 1 8}$} & \multicolumn{2}{c|}{2019} \\
\cline { 2 - 6 } & Media & $\begin{array}{c}\text { \% N sobre la } \\
\text { media }\end{array}$ & Media & $\begin{array}{c}\text { \% N sobre la } \\
\text { media }\end{array}$ & Media & $\begin{array}{c}\text { \% N sobre la } \\
\text { media }\end{array}$ \\
\hline GES1 & 2,0564 & $27 \%$ & 2,5382 & $32 \%$ & 3,0659 & $41 \%$ \\
\hline GES4 & 6,2600 & $68 \%$ & 6,6327 & $73 \%$ & 7,2427 & $64 \%$ \\
\hline GES5 & 7,0427 & $41 \%$ & 5,5350 & $50 \%$ & 8,2727 & $86 \%$ \\
\hline GES9 & 3,2045 & $32 \%$ & 4,6064 & $36 \%$ & 3,7577 & $32 \%$ \\
\hline GES10 & 2,1850 & $45 \%$ & 6,1605 & $50 \%$ & 5,8282 & $45 \%$ \\
\hline GES15 & 2,6600 & $32 \%$ & 1,8473 & $36 \%$ & 1,0277 & $23 \%$ \\
\hline GES19 & 6,3636 & $64 \%$ & 9,5455 & $95 \%$ & 9,1009 & $91 \%$ \\
\hline GES29 & 2,2727 & $23 \%$ & 4,0909 & $41 \%$ & 5,9091 & $59 \%$ \\
\hline GES32 & 6,3064 & $73 \%$ & 3,8136 & $55 \%$ & 4,8032 & $45 \%$ \\
\hline GES33 & 6,3341 & $41 \%$ & 6,8773 & $55 \%$ & 6,3136 & $50 \%$ \\
\hline GES34 & 4,7282 & $55 \%$ & 3,6041 & $50 \%$ & 4,1186 & $55 \%$ \\
\hline GES36 & 4,6182 & $59 \%$ & 2,4673 & $50 \%$ & 2,9614 & $41 \%$ \\
\hline GES37 & 3,9241 & $41 \%$ & 3,8005 & $45 \%$ & 1,4327 & $45 \%$ \\
\hline GES38 & 5,6327 & $50 \%$ & 5,8595 & $45 \%$ & 5,3932 & $50 \%$ \\
\hline GES40 & 3,9759 & $59 \%$ & 2,8964 & $59 \%$ & 3,0091 & $50 \%$ \\
\hline GES43 & 2,5500 & $45 \%$ & 5,1777 & $59 \%$ & 3,1668 & $59 \%$ \\
\hline GES44 & 5,1118 & $41 \%$ & 4,4268 & $50 \%$ & 4,2627 & $50 \%$ \\
\hline GES45 & 7,3968 & $64 \%$ & 7,0273 & $59 \%$ & 6,8095 & $68 \%$ \\
\hline
\end{tabular}

Fuente: Elaboración propia

Los resultados permiten observar un positivo avance en las variables inherentes a la Gestión del Destino en el plano temporal 2017 - 2018, particularmente, en lo referente a la sostenibilidad, los planes de desarrollo, los parafiscales, la financiación de proyectos, la producción de información, los eventos terroristas, la disponibilidad de policía del turismo y las oficinas especializadas en la gestión turística, siendo esta última la más representativa con una variación neta cercana al 30\%, por consiguiente, se infiere la importancia de la profesionalización del sector económico, promovida en gran medida por personal con directa influencia en las zonas geográficas, lo cual propende la mejora en la calidad de vida de los habitantes (Salinas Fernández et al., 2020), así mismo, visiona un enfoque a largo plazo, tal como lo precisa (Budeanu, 2005), no obstante, se identificaron falencias en la seguridad de las empresas turísticas, lo cual afecta notablemente la probabilidad de revisita, teniendo presente características de imagen (Lee et al., 2014) y emociones (Wirtz \& Bateson, 1999).

En relación al plano temporal 2018 - 2019, se pudo denotar un comportamiento en gran medida estable en las diversas variables, puesto que solo se mejoró el 33,33\% de las mismas, a saber, sostenibilidad, planes de desarrollo, producción de información, homicidios, puntos de información turística y delitos cometidos contra los turistas, en tal sentido, se observa que los componentes de índole legal - gubernamental fueron considerados como los focos más representativos (Bălan et al., 2009), lo cual es congruente, teniendo en cuenta la formulación 
prevista por las autoridades departamentales, sin embargo, se observa que lo referente a los rubros presupuestales fueron un obstáculo relevante, afectando notablemente la competitividad del destino (Das \& DiRienzo, 2009)

\subsection{Análisis correlacional}

El análisis correlacional en el marco del desarrollo de la presente investigación tuvo como eje orientador identificar cuantitativamente la relación entre las variables inherentes a la Gestión del Destino en el plano temporal objeto de estudio, del Índice de Competitividad Turística Regional, en tal sentido, en primer lugar, se realizó la prueba de normalidad, particularmente la referente a Kolmogorov - Smirnov, teniendo presente la cantidad de los datos, cuyos resultados se presentan en la Tabla No. 4. Los resultados permiten observar que algunas de las variables tienen un comportamiento acorde a la distribución normal, y por el contrario otras no reflejan tal orientación, siendo esto analizable por medio del $\mathrm{p}-$ valor $>0,05$, por consiguiente, se tuvieron presentes los conceptos de correlación de Pearson y Spearman, los cuales se presentan en la Tabla 4.

Tabla 4. Prueba de normalidad Kolmogorov - Smirnov

\begin{tabular}{|l|r|r|r|}
\hline & \multicolumn{3}{|c|}{ Kolmogorov-Smirnov $^{\mathbf{a}}$} \\
\cline { 2 - 4 } & Estadístico & \multicolumn{1}{|c|}{ gl } & \multicolumn{1}{c|}{ Sig. } \\
\hline GES1 & 0,412 & 65 & 0,000 \\
\hline GES5 & 0,240 & 65 & 0,000 \\
\hline GES9 & 0,135 & 65 & 0,005 \\
\hline GES10 & 0,120 & 65 & 0,022 \\
\hline GES15 & 0,087 & 65 &, $200^{*}$ \\
\hline GES19 & 0,261 & 65 & 0,000 \\
\hline GES29 & 0,510 & 65 & 0,000 \\
\hline GES32 & 0,383 & 65 & 0,000 \\
\hline GES33 & 0,083 & 65 &, $200^{*}$ \\
\hline GES34 & 0,229 & 65 & 0,000 \\
\hline GES36 & 0,084 & 65 &, $200^{*}$ \\
\hline GES37 & 0,096 & 65 &, $200^{*}$ \\
\hline GES38 & 0,109 & 65 & 0,010 \\
\hline GES40 & 0,082 & 65 & 0,052 \\
\hline GES43 & 0,216 & 65 &, $200^{*}$ \\
\hline GES44 & 0,130 & 65 & 0,000 \\
\hline GES45 & 0,176 & 65 & 0,009 \\
\hline & 61000 \\
\hline
\end{tabular}

Fuente: Elaboración propia 
Tabla 5. Correlación de variables: Gestión del Destino

\begin{tabular}{|c|c|c|c|c|c|c|c|c|c|c|c|c|c|c|c|c|c|c|}
\hline & $\begin{array}{c}\text { GE } \\
\text { S1 }\end{array}$ & $\begin{array}{c}\text { GE } \\
\text { S4 }\end{array}$ & $\begin{array}{c}\text { GE } \\
\text { S5 }\end{array}$ & $\begin{array}{c}\text { GE } \\
\text { S9 }\end{array}$ & $\begin{array}{c}\text { GE } \\
\text { S10 }\end{array}$ & $\begin{array}{c}\text { GE } \\
\text { S15 }\end{array}$ & $\begin{array}{c}\text { GE } \\
\text { S19 }\end{array}$ & $\begin{array}{c}\text { GE } \\
\text { S29 }\end{array}$ & $\begin{array}{c}\text { GE } \\
\text { S32 }\end{array}$ & $\begin{array}{c}\text { GE } \\
\text { S33 }\end{array}$ & $\begin{array}{c}\text { GE } \\
\text { S34 }\end{array}$ & $\begin{array}{c}\text { GE } \\
\text { S36 }\end{array}$ & $\begin{array}{l}\text { GE } \\
\text { S37 }\end{array}$ & $\begin{array}{c}\text { GE } \\
\text { S38 }\end{array}$ & $\begin{array}{c}\text { GE } \\
\text { S40 }\end{array}$ & $\begin{array}{c}\text { GE } \\
\text { S43 }\end{array}$ & $\begin{array}{c}\text { GE } \\
\text { S44 }\end{array}$ & $\begin{array}{c}\text { GE } \\
\text { S45 }\end{array}$ \\
\hline $\begin{array}{l}\text { GE } \\
\text { S1 }\end{array}$ & 1,0 & & & & & & & & & & & & & & & & & \\
\hline $\begin{array}{l}\text { GE } \\
\text { S4 }\end{array}$ & 0,2 & 1,0 & & & & & & & & & & & & & & & & \\
\hline $\begin{array}{l}\text { GE } \\
\text { S5 }\end{array}$ & 0,1 & 0,2 & 1,0 & & & & & & & & & & & & & & & \\
\hline $\begin{array}{l}\text { GE } \\
\text { S9 }\end{array}$ & 0,3 & 0,2 & 0,0 & 1,0 & & & & & & & & & & & & & & \\
\hline $\begin{array}{l}\text { GE } \\
\text { S10 }\end{array}$ & 0,3 & 0,3 & 0,1 & 0,6 & 1,0 & & & & & & & & & & & & & \\
\hline $\begin{array}{l}\text { GE } \\
\text { S15 }\end{array}$ & 0,0 & 0,0 & $-0,1$ & 0,2 & 0,0 & 1,0 & & & & & & & & & & & & \\
\hline $\begin{array}{l}\text { GE } \\
\text { S19 }\end{array}$ & 0,2 & 0,2 & 0,0 & 0,3 & 0,3 & 0,0 & 1,0 & & & & & & & & & & & \\
\hline $\begin{array}{l}\text { GE } \\
\text { S29 }\end{array}$ & 0,2 & 0,1 & 0,1 & 0,2 & 0,4 & $-0,3$ & 0,1 & 1,0 & & & & & & & & & & \\
\hline $\begin{array}{l}\text { GE } \\
\text { S32 }\end{array}$ & $-0,3$ & $-0,2$ & 0,0 & $-0,5$ & $-0,5$ & 0,1 & $-0,3$ & $-0,4$ & 1,0 & & & & & & & & & \\
\hline $\begin{array}{l}\text { GE } \\
\text { S33 }\end{array}$ & $-0,1$ & 0,2 & $-0,1$ & 0,1 & 0,0 & 0,2 & 0,0 & $-0,3$ & 0,0 & 1,0 & & & & & & & & \\
\hline $\begin{array}{l}\text { GE } \\
\text { S34 }\end{array}$ & 0,0 & $-0,2$ & $-0,1$ & $-0,1$ & $-0,2$ & 0,1 & $-0,1$ & 0,1 & 0,1 & 0,2 & 1,0 & & & & & & & \\
\hline $\begin{array}{l}\text { GE } \\
\text { S36 }\end{array}$ & $-0,1$ & $-0,2$ & 0,1 & $-0,6$ & $-0,4$ & 0,2 & $-0,4$ & $-0,1$ & 0,5 & 0,0 & 0,0 & 1,0 & & & & & & \\
\hline $\begin{array}{l}\text { GE } \\
\text { S37 }\end{array}$ & 0,1 & $-0,1$ & $-0,4$ & $-0,1$ & $-0,2$ & 0,1 & $-0,2$ & 0,0 & $-0,1$ & 0,3 & 0,3 & 0,3 & 1,0 & & & & & \\
\hline $\begin{array}{l}\text { GE } \\
\text { S38 }\end{array}$ & 0,4 & 0,3 & $-0,1$ & 0,5 & 0,4 & 0,1 & 0,2 & 0,2 & $-0,5$ & 0,2 & 0,2 & $-0,5$ & 0,2 & 1,0 & & & & \\
\hline $\begin{array}{l}\text { GE } \\
\text { S40 }\end{array}$ & $-0,2$ & $-0,3$ & $-0,1$ & $-0,7$ & $-0,5$ & 0,0 & $-0,5$ & $-0,4$ & 0,6 & 0,0 & 0,2 & 0,6 & 0,1 & $-0,6$ & 1,0 & & & \\
\hline $\begin{array}{l}\text { GE } \\
\text { S43 }\end{array}$ & $-0,2$ & 0,0 & $-0,2$ & $-0,1$ & 0,4 & $-0,1$ & 0,0 & $-0,1$ & 0,0 & $-0,1$ & $-0,3$ & 0,0 & 0,1 & $-0,2$ & 0,1 & 1,0 & & \\
\hline $\begin{array}{l}\text { GE } \\
\text { S44 }\end{array}$ & 0,3 & 0,0 & 0,1 & $-0,1$ & 0,0 & $-0,1$ & 0,1 & 0,0 & 0,1 & 0,1 & 0,0 & 0,2 & 0,4 & 0,1 & 0,1 & 0,2 & 1,0 & \\
\hline $\begin{array}{l}\text { GE } \\
\text { S45 }\end{array}$ & $-0,5$ & $-0,3$ & $-0,2$ & $-0,4$ & $-0,4$ & $-0,1$ & $-0,2$ & $-0,3$ & 0,4 & 0,2 & 0,1 & 0,2 & 0,0 & $-0,4$ & 0,4 & 0,1 & $-0,2$ & 1,0 \\
\hline
\end{tabular}

*Los valores sombreados hacen referencia a relaciones significativas

Fuente: Elaboración propia

La Tabla 5 nos indica que existen una considerable cantidad de relaciones moderadas fuertes o débiles (Martínez et al., 2009) entre las variables vinculadas a la Gestión del Destino, particularmente, se destaca lo referente contribución parafiscal - financiamiento de proyectos $(0,6)$, accidentalidad vial - seguridad de empresas turísticas $(0,6)$, accidentalidad vial disponibilidad policial $(0,6)$, disponibilidad policial - seguridad de empresas turísticas $(0,5)$, contribución parafiscal - puntos de información turística (0.5), entre otros, lo cual permite validar la importancia de los canales de comunicación en el desarrollo competitivo de una zona geográfica, tal como lo mencionaba Doolin et al., (2002) en torno a la creación de experiencias y fomento de la imagen del territorio, así mismo, la relevancia de la seguridad en el periodo de estancia (Andrades \& Dimanche, 2017).

Por otro lado, lo inherente a accidentalidad vial - contribución parafiscal $(-0,7)$, puntos de información turística - accidentalidad vial $(-0,6)$, disponibilidad policial - contribución parafiscal $(-0,6)$, seguridad de empresas turísticas - contribución parafiscal $(-0,5)$, seguridad de empresas turísticas - financiamiento de proyectos $(-0,5)$, disponibilidad policial - puntos de información turística $(-0,5)$, accidentalidad vial - financiamiento de proyectos $(-0,5)$, 
accidentalidad vial - oficinas especializadas en gestión turística (-0,5), entre otros, siendo esto vinculable a lo expuesto por Isa et al. (2020) en constructos de intenciones de revisita.

\subsection{Análisis de componentes principales}

La tercera fase de la investigación presenta un análisis factorial de las variables objeto de estudio, con el fin de proponer una serie de dimensiones para la Gestión del Destino, siendo posible por medio de un conjunto de datos de constructos sin correlación lineal, las cuales se denominan componentes principales (Campos et al., 2020; Jolliffe, 2005), en tal sentido, se aplicó el test KMO obteniendo un indicador de 0,6 y la prueba de esfericidad de Barlett con un grado de significancia de 0.000 , lo cual confirma que es factible continuar con el análisis factorial.

En este contexto, la Tabla 6. presenta los autovalores, contemplando la generación de 6 componentes principales, los cuales explican cerca del $70 \%$ de la varianza.

Tabla 6. Varianza total explicada: Componentes principales

\begin{tabular}{|c|c|c|c|c|c|c|c|c|c|}
\hline \multicolumn{9}{|c|}{ Varianza total explicada } \\
\hline \multirow{2}{*}{ Componente } & \multicolumn{3}{|c|}{ Autovalores iniciales } & \multicolumn{2}{c|}{$\begin{array}{c}\text { Sumas de cargas al cuadrado } \\
\text { de la extraccín }\end{array}$} & \multicolumn{2}{|c|}{$\begin{array}{c}\text { Sumas de cargas al cuadrado } \\
\text { de la rotación }\end{array}$} \\
\cline { 2 - 11 } & Total & $\begin{array}{c}\text { \% de } \\
\text { varianza }\end{array}$ & $\begin{array}{c}\text { \% } \\
\text { acumulado }\end{array}$ & Total & $\begin{array}{c}\text { \% de } \\
\text { varianza }\end{array}$ & $\begin{array}{c}\text { \% } \\
\text { acumulado }\end{array}$ & Total & $\begin{array}{c}\text { \% de } \\
\text { varianza }\end{array}$ & $\begin{array}{c}\text { \% } \\
\text { acumulado }\end{array}$ \\
\hline $\mathbf{1}$ & 4,6 & 25,4 & 25,4 & 4,6 & 25,4 & 25,4 & 3,8 & 21,0 & 21,0 \\
\hline $\mathbf{2}$ & 2,1 & 11,5 & 36,8 & 2,1 & 11,5 & 36,8 & 2,1 & 11,8 & 32,7 \\
\hline $\mathbf{3}$ & 1,7 & 9,5 & 46,3 & 1,7 & 9,5 & 46,3 & 2,0 & 11,1 & 43,8 \\
\hline $\mathbf{4}$ & 1,6 & 8,8 & 55,1 & 1,6 & 8,8 & 55,1 & 1,6 & 8,9 & 52,7 \\
\hline $\mathbf{5}$ & 1,4 & 7,8 & 62,9 & 1,4 & 7,8 & 62,9 & 1,5 & 8,4 & 61,1 \\
\hline $\mathbf{6}$ & 1,2 & 6,6 & 69,5 & 1,2 & 6,6 & 69,5 & 1,5 & 8,3 & 69,5 \\
\hline $\mathbf{7}$ & 0,9 & 5,3 & 74,7 & & & & & & \\
\hline $\mathbf{8}$ & 0,8 & 4,5 & 79,2 & & & & & & \\
\hline $\mathbf{9}$ & 0,7 & 3,9 & 83,1 & & & & & & \\
\hline $\mathbf{1 0}$ & 0,7 & 3,7 & 86,8 & & & & & & \\
\hline $\mathbf{1 1}$ & 0,5 & 2,9 & 89,7 & & & & & & \\
\hline $\mathbf{1 2}$ & 0,4 & 2,5 & 92,1 & & & & & & \\
\hline $\mathbf{1 3}$ & 0,4 & 2,2 & 94,4 & & & & & & \\
\hline $\mathbf{1 4}$ & 0,3 & 1,9 & 96,3 & & & & & & \\
\hline $\mathbf{1 5}$ & 0,3 & 1,6 & 97,8 & & & & & & \\
\hline $\mathbf{1 6}$ & 0,2 & 1,0 & 98,8 & & & & & & \\
\hline $\mathbf{1 7}$ & 0,1 & 0,7 & 99,5 & & & & & & \\
\hline $\mathbf{1 8}$ & 0,1 & 0,5 & 100,0 & & & & & & \\
\hline & & & & & & & & \\
\hline
\end{tabular}

Fuente: Elaboración propia 
Complementariamente, la Tabla 7 presenta la matriz de componente rotado, cuyos resultados son los siguientes:

$\checkmark$ Componente 1: La dimensión se encuentra conformada por el financiamiento de proyectos, las oficinas especializadas en gestión turística, la producción de información estadística de viajes y turismo, y los puntos de información turística, siendo este último constructo el más representativo.

$\checkmark$ Componente 2: La dimensión se compone por la seguridad de empresas turísticas, la accidentalidad vial, los delitos cometidos contra turistas y los eventos terroristas, siendo este último constructo el más representativo.

$\checkmark$ Componente 3: La dimensión contempla los destinos certificados en sostenibilidad, los homicidios, la disponibilidad policial, la disponibilidad de policía de turismo y el presupuesto regionalizado para el turismo, siendo este último constructo el más representativo.

$\checkmark$ Componente 4: La dimensión contempla exclusivamente lo referente al retorno a la contribución parafiscal.

$\checkmark$ Componente 5: La dimensión integra los planes de desarrollo turístico y el turismo en los planes de desarrollo, siendo este último constructo el más representativo.

$\checkmark$ Componente 6: La dimensión estudia la contribución parafiscal y el presupuesto de inversión destinado al turismo, siendo este último constructo el más representativo.

Tabla 7. Matriz de componente rotado

\begin{tabular}{|l|c|c|c|c|c|c|}
\hline & \multicolumn{7}{|c|}{ Componente } \\
\cline { 2 - 7 } & 1 & 2 & 3 & 4 & 5 & 6 \\
\hline GES1 & 0,246 & $-0,422$ & 0,468 & $-0,131$ & 0,284 & 0,189 \\
\hline GES4 & 0,272 & 0,084 & 0,265 & 0,141 & 0,474 & 0,341 \\
\hline GES5 & $-0,104$ & $-0,108$ & $-0,062$ & $-0,035$ & $\mathbf{0 , 8 2 0}$ & $-0,079$ \\
\hline GES9 & 0,500 & $-0,309$ & $-0,191$ & $-0,156$ & 0,077 & 0,556 \\
\hline GES10 & 0,519 & $-0,411$ & $-0,009$ & 0,431 & 0,055 & 0,423 \\
\hline GES15 & $-0,259$ & 0,269 & 0,008 & $-0,030$ & $-0,042$ & $\mathbf{0 , 7 6 5}$ \\
\hline GES19 & 0,688 & 0,211 & $-0,024$ & 0,085 & 0,097 & $-0,134$ \\
\hline GES29 & 0,189 & $-0,758$ & 0,039 & $-0,051$ & $-0,008$ & $-0,071$ \\
\hline GES32 & $-0,691$ & 0,353 & $-0,174$ & $-0,071$ & 0,098 & $-0,087$ \\
\hline GES33 & 0,173 & $\mathbf{0 , 6 8 1}$ & 0,286 & $-0,125$ & $-0,009$ & 0,229 \\
\hline GES34 & $-0,043$ & $-0,011$ & 0,179 & $-0,683$ & $-0,299$ & $-0,005$ \\
\hline GES36 & $-0,826$ & 0,088 & 0,266 & 0,028 & 0,064 & 0,046 \\
\hline GES37 & $-0,121$ & 0,012 & 0,787 & $-0,168$ & $-0,372$ & 0,133 \\
\hline GES38 & $\mathbf{0 , 6 9 8}$ & $-0,119$ & 0,269 & $-0,272$ & 0,008 & 0,263 \\
\hline GES40 & $-0,872$ & 0,136 & $-0,029$ & $-0,046$ & $-0,103$ & 0,063 \\
\hline GES43 & $-0,052$ & 0,016 & 0,164 & $\mathbf{0 , 8 4 6}$ & $-0,281$ & $-0,079$ \\
\hline GES44 & $-0,020$ & 0,067 & $\mathbf{0 , 7 9 7}$ & 0,147 & 0,199 & $-0,200$ \\
\hline GES45 & $-0,282$ & 0,576 & $-0,298$ & 0,093 & $-0,387$ & $-0,215$ \\
\hline
\end{tabular}

Fuente: Elaboración propia 


\section{Conclusiones}

El boom que ha vivido el sector turístico en las últimas décadas ha motivado a las autoridades gubernamentales a enfocarse en los factores que podrían dar a sus países y territorios una ventaja competitiva. Con ello, la competitividad turística se convierte en una cuestión clave para el desarrollo del turismo.

La competitividad turística puede entenderse desde tres niveles: (1) el nivel del país (competitividad de la producción turística), (2) el nivel del destino o (3) el nivel de la empresa turística. Desde un enfoque de desarrollo sostenible, la competitividad de un país o un destino turístico está relacionada con su capacidad para conseguir dos tipos de objetivos: satisfacción y productividad. La satisfacción es la capacidad del destino de entregar valor a los turistas, mientras que la productividad se relaciona directamente con el desarrollo humano y determina en qué medida se usan eficazmente los recursos.

La subjetividad, la multiplicidad de variables que intervienen en su evaluación y la dificultad de dibujar los límites entre un destino y su entorno dificultan la formulación de medidas reales de competitividad turística. Aun así, se ha desarrollado una diversidad de modelos para medir la competitividad de un destino. Entre los modelos académicos de competitividad turística resalta el de Ritchie y Crouch (2010), que se compone de siete elementos: el entorno global (macro); el entorno competitivo (micro); los recursos básicos y los atractivos; factores y recursos de apoyo; política, planificación y desarrollo de los destinos; gestión del destino; calificación y amplificación de los factores determinantes. Otro modelo muy difundido es el Índice de Competitividad de Viajes y Turismo (TTCI), elaborado por el FEM. Sin embargo, este índice es una herramienta de benchmarking para la formulación de políticas y estrategias para la sustentabilidad turística, más que una medida real de la competitividad turística.

En el caso de Colombia, Cotelco y la Fundación Universitaria Cafam publican el Índice de Competitividad Turística Regional de Colombia (ICTRC) para todos los departamentos del país. El ICTRC ha permitido aplicar un sistema único de medición de la competitividad turística en todo el país, para el beneficio de los gestores públicos, la academia y la sociedad en general (CPTUR, 2016).

La competitividad turística es un constructo ampliamente polisémico, el cual ha sido objeto de estudio por diversos investigadores alrededor de todo el mundo, en tal sentido, la presente investigación indago exclusivamente la variable concerniente a la gestión del destino en el plano temporal 2017 - 2019, teniendo presente los resultados del Índice de Competitividad Turística Regional de Colombia (ICTRC) desarrollado por el Centro de Pensamiento Turístico, permitiendo identificar positivos avances en torno al turismo en los planes de desarrollo y oficinas especializadas en gestión turística, lo cual en gran medida se alinea con lo expuesto por Rodríguez-Díaz \& Pulido-Fernández (2020) frente a la relevancia de la actividad económica en la sociedad actual. 
Las variables inherentemente vinculadas a la Gestión del Destino presentan niveles de correlación representativos, sean moderadamente fuertes o débiles (Martínez et al., 2009), lo cual permite afirmar que la presencia al alza o a la baja de un determinado indicador posibilita elementos a favor o en contra de otro, en otros términos, las zonas geográficas generadoras de ofertas turísticas no deben analizar aisladamente los diversos constructos, debido a que el turista reconoce un determinado sistema, y el correcto desarrollo del mismo permitirá en gran medida un voz a voz positivo y a gran escala tasas representativas de revisita y mejora en la calidad de vida los habitantes (Salinas Fernández et al., 2020).

El análisis de componentes principales permitió identificar que los focos principales de operación en torno a la gestión del destino se orientan hacia la información turística, los eventos terroristas, los niveles de presupuestales, el retorno a la contribución parafiscal y la inclusión del turismo en los planes de desarrollo. Por consiguiente, se infiere que las entidades gubernamentales deberán formular, implementar y validar procesos a nivel macro y micro en los constructos referidos, con el fin de impactar considerablemente la competitividad en las zonas geográficas.

Las futuras de líneas de investigación referentes al presente estudio consideran aplicar análisis paralelos a muestras más grandes de zonas geográficas, con el propósito de contrastar las conclusiones formuladas, así mismo, considerar diferentes variables vinculables a la competitividad turística, en el marco de consolidar una serie de sugerencias válidas para los entes gubernamentales.

\section{Referencias}

Acerenza, M.A. (2009): Competitividad de los Destinos Turísticos. México: Trillas.

Andrades, L., \& Dimanche, F. (2017). Destination competitiveness and tourism development in Russia: Issues and challenges. Tourism Management, 62, 360-376. https://doi.org/10.1016/j.tourman.2017.05.008

Azzopardi, E., \& Nash, R. (2017). A Review of Crouch and Ritchie's, Heath's, and Dwyer and Kim's Models of Tourism Competitiveness. Tourism analysis, 22(2), 247-254. https://doi.org/10.3727/108354217X14888192562483

Bălan, D., Virgil, B., \& C, V. (2009). Travel And Tourism Competitiveness Of The Worldâ $€^{\mathrm{TM}} \mathrm{S}$ Top Tourism Destinations: An Exploratory Assessment. Annales Universitatis Apulensis Series Oeconomica, 2(11), 1-43.

Benavides, G., \& Venegas, S. (2020). Una aproximación a la competitividad, las tendencias y la política pública en el turismo colombiano. http://hdl.handle.net/20.500.12010/9403

Brandão, M., Joia, L. A., \& do Canto Cavalheiro, G. M. (2019). Towards a Smart Destination 
Development Model: Promoting Environmental, Economic, Socio-Cultural and Political Values. In Setting Foundations for the Creation of Public Value in Smart Cities (pp. 137161). Springer, Cham. https://doi.org/10.1007/978-3-319-98953-2_6

Budeanu, A. (2005). Impacts and responsibilities for sustainable tourism: A tour operator's perspective. Journal of Cleaner Production, 13 (2), 89-97. https://doi.org/10.1016/j.jclepro.2003.12.024

Campos, C. I. de, Pitombo, C. S., Delhomme, P., \& Quintanilha, J. A. (2020). Comparative analysis of data reduction techniques for questionnaire validation using self-reported driver behaviors. Journal of Safety Research, 73, 133-142. https://doi.org/10.1016/j.jsr.2020.02.004

Cavalheiro, M. B., Joia, L. A., \& Cavalheiro, G. M. do C. (2020). Towards a Smart Tourism Destination Development Model: Promoting Environmental, Economic, Socio-cultural and Political Values. Tourism Planning and Development, 17(3), 237-259. https://doi.org/10.1080/21568316.2019.1597763

Centro de Pensamiento Turístico de Colombia - CPTUR. (2016). Índice de Competitividad Turística Regional de Colombia - ICTRC 2016. Bogotá. Cotelco- Unicafama

Corzo, D. (2013). Valoración del grado de competitividad del sector turístico en la ciudad de Bucaramanga. Aplicación del sistema de indicadores definido por el Ministerio de Comercio, Industria y Turismo. Universidad Autónoma de Bucaramanga. https://doi.org/10.13140/RG.2.2.22349.05605

Croes, R., Ridderstaat, J., \& Shapoval, V. (2020). Extending tourism competitiveness to human development. Annals of Tourism Research, 80, 102825. https://doi.org/10.1016/j.annals.2019.102825

Croes, R., \& Kubickova, M. (2013). From potential to ability to compete: Towards a performance-based tourism competitiveness index. Journal of Destination Marketing \& Management, 2(3), 146-154. https://doi.org/10.1016/j.jdmm.2013.07.002

Crouch, G.I. (2011). Destination competitiveness: an analysis of determinant attributes, Journal of Travel Research, n. ${ }^{\circ} 50$ (1), (2011), pp. 27-45. https://doi.org/10.1177/0047287510362776

Das, J., \& Dirienzo, C. E. (2012). Tourism competitiveness and the role of fractionalization. International Journal of Tourism Research, 14(3), 285-297. https://doi.org/10.1002/jtr.866

Das, J., \& DiRienzo, C. E. (2009). Global tourism competitiveness and freedom of the press: A nonlinear relationship. Journal of Travel Research, 47(4), 470-479. 
https://doi.org/10.1177/0047287508326535

Dogru, T., Suess, C., \& Sirakaya-Turk, E. (2020). Why Do Some Countries Prosper More in Tourism than Others? Global Competitiveness of Tourism Development. Journal of Hospitality \& Tourism Research. https://doi.org/10.1177/1096348020911706

Doolin, B., Burgess, L., \& Cooper, J. (2002). Evaluating the use of the Web for tourism marketing: A case study from New Zealand. Tourism Management, 23(5), 557-561. https://doi.org/10.1016/S0261-5177(02)00014-6

González, R. C., \& Mendieta, M. D. (2009). Reflexiones sobre la conceptualización de la competitividad de destinos turísticos. Cuadernos de turismo, (23), 111-128. https://revistas.um.es/turismo/article/view/70201

Garcés, Y., Sarmiento, Y., \& Paneca, Y. (2018). La competitividad turística: su medición y factores determinantes. Caribeña de Ciencias Sociales, (junio). En línea: https://www.eumed.net/rev/caribe/2018/06/competitividad-turistica.html

Gooroochurn, N., Sugiyarto G., (2004), Measuring competitiveness, Discussion Paper, TTRI, Nottingham University Business School. University of Nottingham

Hermawati, A. (2020). Transglobal leadership approach to sustainable tourism competitiveness at tourism sector-engaged MSMEs through integrated human resource performance and responsible marketing. International Journal of Tourism Cities. https://doi.org/10.1108/IJTC-06-2019-0085

Hernández, R., Fernández, C., \& Baptista, P. (2006). Metodología de la investigación. Iztapalapa. México DF: Mc Graw Hill.

Hong, W. C. (2009). Global competitiveness measurement for the tourism sector. Current issues in tourism, 12(2), 105-132. https://doi.org/10.1080/13683500802596359

Isa, S. M., Ariyanto, H. H., \& Kiumarsi, S. (2020). The effect of place attachment on visitors' revisit intentions: evidence from Batam. Tourism Geographies, 22(1), 51-82. https://doi.org/10.1080/14616688.2019.1618902

Jolliffe, I. (2005). Principal Component Analysis. In Encyclopedia of Statistics in Behavioral Science (Vol. 3, pp. 1580-1584). John Wiley \& Sons, Ltd. https://doi.org/10.1002/0470013192.bsa501

Krstic, B., Jovanovic, S., Jankovic-Milic, V., \& Stanisic, T. (2016). Examination of travel and tourism competitiveness contribution to national economy competitiveness of sub-Saharan Africa countries. Development Southern Africa, 33(4), 470-485. https://doi.org/10.1080/0376835X.2016.1179103 
Knežević Cvelbar, L., Dwyer, L., Koman, M., \& Mihalič, T. (2016). Drivers of destination competitiveness in tourism: a global investigation. Journal of Travel Research, 55(8), 1041-1050. https://doi.org/10.1177/0047287515617299

Kolosinska, M., Petrashchak, O., Kolosinskyi, I., \& Katana, A. (2018). Tourism sector in transition economy on example of Ukraine: determinants of competitiveness. GeoJournal of Tourism and Geosites, 21 (1), 239-252.

Kubickova, M., \& Lee, S. H. (2018). Cuba today: An overview of tourism competitiveness. $\begin{array}{llll}\text { Tourism Planning \& } \quad \text { Development, } & \text { 15(3), }\end{array}$ https://doi.org/10.1080/21568316.2017.1410496

Lee, J., Kao, H.-A., \& Yang, S. (2014). Service Innovation and Smart Analytics for Industry 4.0 and Big Data Environment. Procedia CIRP, 16, 3-8. https://doi.org/10.1016/j.procir.2014.02.001

Maráková, V., Dyr, T., \& Wolak-Tuzimek, A. (2016). Factors of tourism's competitiveness in European union countries. Economics and Management. https://dspace.tul.cz/handle/15240/18194

Martínez, R., Tuya, L., Martínez, M., Pérez, A., \& Cánovas, A. (2009). El coeficiente de correlacion de los rangos de spearman caracterizacion. Revista Habanera de Ciencias Medicas, 8(2).

Melián-González, A., García-Falcón, J., M., (2003), Competitive potential of tourism in destinations, Annals of Tourism Research, v. 30, n. 3, pp.720-740. https://doi.org/10.1016/S0160-7383(03)00047-1

Mihalic, T., \& Aramberri, J. (2015). Myths of top tourism countries, tourism contribution and competitiveness. Tourism Review. Vol. 70 No. 4, pp. 276-288. https://doi.org/10.1108/TR08-2014-0048

Ministerio de Comercio, Industria y Turismo (MINCIT). (2008). Sistema de Indicadores de Competitividad para el Turismo Colombiano (versión preliminar). Bogotá: MINCIT.

Mustafa, H., Omar, B., \& Mukhiar, S. N. S. (2020). Measuring destination competitiveness: an importance-performance analysis (IPA) of six top island destinations in South East Asia. Asia Pacific Journal of Tourism Research, 25(3), 223-243. https://doi.org/10.1080/10941665.2019.1687534

Nazmfar, H., Eshghei, A., Alavi, S., \& Pourmoradian, S. (2019). Analysis of travel and tourism competitiveness index in middle-east countries. Asia Pacific Journal of Tourism Research, 24(6), 501-513. https://doi.org/10.1080/10941665.2019.1590428 
Ortiz, F.I., Robles, V.H. \& Guzmá, A. (2018). Análisis teórico de los modelos de competitividad de destinos turísticos. Un acercamiento para la competitividad de los destinos turísticos mexicanos, International Journal of Scientific Management and Tourism, 4-3. pp. 195-221.

Peña, D. (2002): Análisis de Datos Multivariantes. Madrid: McGraw-Hill/Interamericana de España, S.A.U. Aravaca.

Perles, J., Ramón, A. \& Sevilla, M. (2014). La cuota de mercado como indicador de competitividad en los destinos turísticos: sentido y limitaciones. Cuadernos de Turismo, pp. 265-285. https://revistas.um.es/turismo/article/view/203151

Pérez, V. E., Peral, F. J. B., González, M., Casas, F. M. G., Lozano-Oyola, M., García, F. P., \& Fernández, R. C. (2009). Evaluación de la sostenibilidad del turismo rural mediante indicadores sintéticos. Investigación operacional, 30(1), 40-51. https://rua.ua.es/dspace/bitstream/10045/92533/1/2016_Perles-Ribes_Canelobre.pdf

Perles Ribes, J. F. (2016). La competitividad turística: una perspectiva empresarial y territorial. Revista del Instituto Alicantino de Cultura Juan Gil-Albert, 66, 205-2015

Porter, M. E. (1985). Ventaja competitiva: creación y sostenimiento de un desempeño superior. México: Grupo editorial patria México, CECSA.

Rodríguez-Díaz, B., \& Pulido-Fernández, J. I. (2020). Analysis of the Worth of the Weights in a new Travel and Tourism Competitiveness Index. Journal of Travel Research. https://doi.org/10.1177/0047287519899982

Rodríguez-Díaz, B., \& Pulido-Fernández, J. I. (2020a). Sustainability as a key factor in tourism competitiveness: a global analysis. Sustainability, $12(1), \quad 51$. https://doi.org/10.3390/su12010051

Rodríguez, J. M., Rubi, L., Alonso, M., \& Celerin, M. (2016). Aplicación de un modelo cuantitativo objetivo para la medición de la competitividad de los destinos turísticos. Cuadernos de turismo, (38), 415-430. https://doi.org/10.6018/turismo.38.271501

Romão, J. (2020). Variety, smart specialization and tourism competitiveness. Applied Sciences (Switzerland), 12(14), 1-13. https://doi.org/10.3390/su12145765

Salinas Fernández, J. A., Serdeira Azevedo, P., Martín Martín, J. M., \& Rodríguez Martín, J. A. (2020). Determinants of tourism destination competitiveness in the countries most visited by international tourists: Proposal of a synthetic index. Tourism Management Perspectives, 33(October 2018), 100582. https://doi.org/10.1016/j.tmp.2019.100582 
Serrano, A. M., Montoya, L. A. R., \& Cazares, I. (2018). Análisis de la sostenibilidad y competitividad turística en Colombia. Gestión y Ambiente, 21(1), 99-109.

Varela, R. V., \& Olarte, T. O. (2018). Análisis de la competitividad del sector turístico del área metropolitana de Bucaramanga (Colombia). Anuario Turismo y Sociedad, (23), 199-211.

Wang, X., \& Liu, D. (2020). The Coupling Coordination Relationship between Tourism Competitiveness and Economic Growth of Developing Countries. Sustainability, 12(6), 2350. https://doi.org/10.3390/su12062350

Wan, J., Yan, J., Wang, X., Liu, Z., Wang, H., \& Wang, T. (2019). Spatial-Temporal Pattern and Its Influencing Factors on Urban Tourism Competitiveness in City Agglomerations Across the Guanzhong Plain. Sustainability, 11(23), 6743. https://doi.org/10.3390/su11236743

Webster, C., \& Ivanov, S. (2014). Transforming competitiveness into economic benefits: Does tourism stimulate economic growth in more competitive destinations?. Tourism Management, 40, 137-140. https://doi.org/10.1016/j.tourman.2013.06.003

Wirtz, J., \& Bateson, J. (1999). Consumer satisfaction with services integrating the environment perspective in services marketing. Journal of Business Research, 2963(97), 55-66. http://linkinghub.elsevier.com/retrieve/pii/S0148296397001781\%5Cnpapers3://publicati on/uuid/09089BAF-45A7-49F2-B3D8-D549D7D62936

Zuñiga, A., \& Castillo, M. (2012). Turismo en Colombia: resultados del sector (2007-2010). Magazine Empresarial, $8(15)$ 67-73. https://repository.usc.edu.co/handle/20.500.12421/2269 